\title{
Multiplicity of human scent signature
}

Petr Doležal ${ }^{1 *}$ (D) Kenneth G. Furton², Jana Lněničková ${ }^{3}$, Pavlína Kyjaková ${ }^{4}$, Veronika Škeříková', Irena Valterová ${ }^{4}$, Ludvík Pinc ${ }^{3}$ and Štěpán Urban ${ }^{1}$

\begin{abstract}
Background: In this paper, the term "human scent signature" refers to a group of chemical compounds in a human scent trace that are in certain relative concentrations that make an unambiguous identification of persons possible. At the present time, the scent identification of persons is performed using specially trained canines. However, the scent identification mechanism as well as the molecular composition of the human scent signature is entirely unrecognized. Delimitations of the group of chemical compounds according to their volatilities that specially trained canines use for the scent identification of persons were initially the main aim of this study.

Methods: Eleven volunteer scent samples were split into three groups of chemical compounds, i.e., into three disjointed scent fractions with different volatilities of the compounds via preparative gas chromatography. The human scent signatures were searched in these separated scent fractions using six specially trained canines (female German Shepherds).
\end{abstract}

Results: The canines were able to identify individual persons successfully using different scent fractions. From this fact, it follows that one scent trace can contain more than only one group of scent compounds that can be used for scent identification by the trained canines. This observed phenomenon was named as "the multiplicity of the human scent signature" in this study. The scent identification line-up procedure provided the following results: The poorly volatile fraction resulted in an $85 \%$ success rate in the scent identification procedure. The middle fraction resulted in a $58 \%$ success rate, and the highly volatile fraction resulted in a $30 \%$ success rate.

Conclusions: In this study, it was found that the human scent sample contains more than one group of scent compounds; according to which the trained canines are able to identify human individuals. It means that the human scent sample contains more than only one scent signature. The authors of this research believe that results of this study will open a broad discussion about the multiplicity of the human scent signature, primary scent molecules, optimal canine training, and in general, the scent identification method in many countries.

Keywords: Forensic science, Forensic odorology, Forensic olfactory identifications, Preparative gas chromatography, Scent identification line-up, Human scent signature

\section{Background}

Identifying the scents of individual persons using specially trained canines is a well-known forensic method (Settle et al. 1994; Schoon 1996; Schoon 2005) frequently used in criminal investigations. The results of these identifications are generally understood to be subjective and are legally accepted as corroborating evidence (Marchal et al. 2016; Tomaszewski and Girdwoyn 2006) only in some countries (the USA, Spain, France, Russia, Czechia, Poland, Slovakia, etc.). Hence, the

\footnotetext{
*Correspondence: dolezald@vscht.cz; petr.dolezal21@seznam.cz

${ }^{1}$ Department of Analytical Chemistry, University of Chemistry and

Technology Prague, Prague 6, Czech Republic

Full list of author information is available at the end of the article
}

development of an objective, instrumental method that will enable comparative scent identifications is highly desirable. Clearly, such an analytical technique would be more credible from forensic and judicial perspectives. To develop such an instrumental method, profound chemical understanding of the human scent signature is absolutely necessary. However, this knowledge has so far been lacking.

From a chemical perspective, the human scent is a complicated mixture of several thousand compounds that significantly differ (Curran et al. 2010; Curran et al. 2005; Doležal et al. 2017; Gallagher et al. 2008) in concentration and chemical properties. The human scent 
can usually be classified into primary, secondary, and tertiary categories (Curran et al. 2005). The primary scent category includes compounds that are assumed to be genetically conditioned. Their relative concentrations are approximately stable over time regardless of environmental factors, diet, weather, humidity, visceral state, emotional state, illness, menstrual cycle, medication, etc. On the other hand, the skin scent compounds, whose amounts depend on these internal and external factors, are in the secondary category. Finally, tertiary scents originate from the external environment, e.g., from cosmetics used, scents of the workplace, gasoline, smoking, scents of other people, and domestic animals.

From the above definitions of the scent categories, the active human scent signature should comprise the primary scent compounds. However, these primary molecules have not yet been unambiguously recognized; nevertheless, many papers (Curran et al. 2010; Curran et al. 2005; Gallagher et al. 2008; Prada et al. 2015) assume that the human scent signature is formed primarily by volatile organic compounds (VOC). In principle, the designation "volatile organic compounds" does not differentiate between highly or poorly volatile compounds. However, the majority of previous chemical analyses of human scent samples (Cui et al. 2009; Curran et al. 2010; Curran et al. 2005; Kusano et al. 2011; Pandey and Kim 2011) implicitly presumed that the highly (and perhaps also moderately) volatile compounds are the most important for human scent signatures. This assumption follows from the analytical chemistry methods that are typically used in these chemical scent analyses. Headspace solid-phase microextraction gas chromatography/ mass spectrometry (HS-SPME-GC/MS), which was frequently used for the chemical analyses of human scent samples (Cui et al. 2009; Curran et al. 2010; Curran et al. 2005; Kusano et al. 2011; Pandey and Kim 2011), discriminates against poorly volatile compounds (Pandey and Kim 2011). Nevertheless, from a forensic point of view, these poorly volatile compounds can be extremely important since the collection of a scent trace from a crime scene can be delayed by several days to a couple of weeks, but the scent signature is still recognizable by trained canines.

The essential aim of this study was to specify the group of chemical compounds that specially trained canines use for the scent identification of persons. Therefore, scent samples from 11 volunteers were split into three disjointed fractions using a gas chromatograph connected with a preparative fraction collector (GC-PFC). These scent fractions were subjected to a scent identification line-up using specially trained canines to determine which fraction contains the active scent signature. The volunteers were chosen to be the same type, therefore the same sex, age (approximately 22 years old), and the same European ethnicity, to eliminate possible effects of the "class identification" in our identification line-up procedure.

\section{Methods \\ Preparation of human scent samples}

The scent samples were taken from the volunteer's palms using 40 chemical cleaned glass beads as a sorbent (Bernier et al. 1999; Doležal et al. 2017). This sorption material (soda-lime glass, $4.86 \pm 0.17 \mathrm{~mm}$ in diameter) was used for scent collection because, compared with the fabrics typically used (sterile gauze, gauze pads, or a blend of cotton, rayon, polyester, wool, etc.), it can be cleaned significantly better (Prada et al. 2010). The glass beads were thoroughly purified first in a mixture of dichromate-sulfuric acid and then in ultrapure water, ethanol (>99.8\%, PENTA, Ltd., Czech Republic), and hexane ( $\geq 97.0 \%$, Sigma-Aldrich, Merck, Saint Louis, USA), and finally, they were dried in an oven at $210^{\circ} \mathrm{C}$ for $5 \mathrm{~h}$. The purified beads were stored in a desiccator until sampling. The purity of the cleaned glass beads was tested by GC/MS. The purification details are described in our previous study (Doležal et al. 2017).

Female volunteers were chosen for this primary study since their scents are usually more recognizable by the specially trained canines than male scents (Jezierski et al. 2012).

First, the volunteers intensively washed their palms with a special soap (non-perfumed, Amadeus Neutral soap, CORMEN, Czech Republic) and warm water. The volunteers' palms were then allowed to dry in the air for $10 \mathrm{~min}$. Subsequently, those volunteers rolled 40 chemically purified beads between their open palms for $10 \mathrm{~min}$. After that, samples were extracted from the beads with the adsorbed scent two times with $0.7 \mathrm{ml}$ hexane. The extracted samples free evaporated from a volume of $1400 \mu \mathrm{l}$ to $200 \mu \mathrm{l}$. These scent samples were stored in a refrigerator (below $7{ }^{\circ} \mathrm{C}$, $44.6^{\circ} \mathrm{F}$ or $280.2 \mathrm{~K}$ ) for up to 1 week before the chromatographic experiments were performed.

\section{Preparations of scent fractions using a preparative GC}

An Agilent 6890 GC/FID with an automatic Preparative Fraction Collector (GC-PFC, GERSTEL GmbH \& Co.KG, Mülheim an der Ruhr, Germany) was used for the GC-preparation of the scent fractions. The preparative column used was an Rxi ${ }^{\circ}-5 \mathrm{~ms}$ (length $30 \mathrm{~m}$, ID $0.53 \mathrm{~mm}$, $\mathrm{d}_{\mathrm{f}} 1.5 \mu \mathrm{m}$; Restek Corporation, Bellefonte, USA) with $\mathrm{He}$ (5.5) as the carrier gas (constant flow rate: $6.0 \mathrm{ml} / \mathrm{min}$ ). The total run time was $46 \mathrm{~min}$. The PTV (programmable temperature vaporization) injection was performed in the splitless mode (SSL). The GC temperature program and the settings of the SSL-PTV and the fraction collector (the heated box) are listed in Table 1.

All the human scent samples were split into three fractions (see Table 2 and Fig. 1). The first fraction (to decanoic acid) are VOCs, the second fraction contains 
Table 1 GC-PFC parameters

\begin{tabular}{lcll}
\hline Temperature $1\left({ }^{\circ} \mathrm{C}\right)$ & Rate $\left({ }^{\circ} \mathrm{C} / \mathrm{min}\right)$ & Temperature $2\left({ }^{\circ} \mathrm{C}\right)$ & Hold $(\mathrm{min})$ \\
\hline GC temperature program & - & 110 & 2 \\
110 & 15 & 320 & 30 \\
110 & & \\
PTV temperature program & 0 & 0.1 \\
0 & 700 & 330 & 4 \\
0 & 100 & 250 & 41.9 \\
330 &
\end{tabular}

moderately volatile compounds, and the third "after squalene" fraction contains poorly volatile compounds such as fatty acid esters (Doležal et al. 2017). The retention indices were calibrated using n-alkanes (C7-C40, Sigma-Aldrich, Merck, Germany). Seven runs of analyses with 5 - $\mu$ l injection volumes were carried out for each sample, which means that a total volume of $35 \mu \mathrm{l}$ was injected for each sample. The Grob Test Mix (Sigma-Aldrich, Merck, Germany) was injected between the scent samples of different volunteers, and the efficacies of the individual peaks were monitored.

The fractions were captured in $1-\mu l$ coiled glass traps that were immersed in the cooling box (at a temperature of $-50{ }^{\circ} \mathrm{C}$, cooled by liquid N2). The glass traps were stored in cleaned jars with twist-off lids, which afterwards were used for the starting scents (SS) in the line-up analyses with the trained canines.

\section{Human scent identification by specially trained canines}

All 33 fractions of human scent samples were obtained from the 11 female volunteers. These fractioned scent samples were subjected to scent identification line-ups as starting scents (Vyplelová et al. 2014) using six canines (female German Shepherds) that were specially trained for the scent identification line-up method. These starting scent samples were prepared from the volunteer's scent samples, which were obtained either from the 1st fraction (highly volatile) or from the 2nd fraction (moderately volatile) or from the 3rd fraction (poorly volatile compounds). The starting samples which split into these three fractions were compared with the

Table 2 Fraction retention times and corresponding retention indices (RI)

\begin{tabular}{lll}
\hline Fraction & Retention index intervals (RI) & Time intervals (min) \\
\hline 1st fraction & $900-1300^{\mathrm{a}}$ & $3.4-7.4$ \\
2nd fraction & $1300-2900^{\mathrm{b}}$ & $7.4-17.8$ \\
3rd fraction & $2900-4000^{\mathrm{c}}$ & $17.8-45.9$ \\
\hline
\end{tabular}

${ }^{\mathrm{a} U p}$ to decanoic acid (not included). ${ }^{\mathrm{b}}$ From decanoic acid to squalene. ${ }^{\mathrm{c}}$ After squalene "full" (i.e., non-fractioned) scent samples in the line-up (see diagram in Fig. 2, lower right side) where the target scent (TS) consisted of only one sample, and the remaining samples were distracting scent (DS) samples. These scent samples (DS) were collected from other volunteers on sterilized fabric sorbents (cotton-based) that are ordinarily used by the Czech Republic's police. To obtain reliable results, the distracting scent samples were taken from volunteers of the same sex and similar age to avoid the "odd-man out" effect (Schoon and Haak 2002).

Before the line-up scent identification, all the canines were subjected to attractivity tests (AT) (see Fig. 2). The sense and details of the attractivity test as well as the standard human scent identification line-up procedure are described in many studies (e.g., (Pinc et al. 2011; Santariová et al. 2016; Schoon and Haak 2002; Vyplelová et al. 2014).

The canines were allowed to sniff the fractioned starting scent samples (SS) and then were sent to search for the target scent (TS) in the line-up (Fig. 2). The canines indicated an identified target scent (TS) by sitting or lying down. All the scent identifications were performed consistently in a double-blind routine, which means that neither the dog handler nor anyone else in the experimental room had any information on the position of the target scent (TS) in the line-up. Each of the scent fractions was tested by the canine three times. The positions of the scent samples (TS and DS) in the lines were randomly (using dice) interchanged by another assistant.

If the canine did not identify the starting scent (random selected fraction from the scent sample of person 1) with the target scent in the line-up, the next fraction (second or third) from the same person was also tested by the same canine. For the opposite case, this canine was replaced by another canine and this one tested the next scent fractions from person 1 (see Tables 3 and 4) to avoid misleading identifications due to the memory effect (Schoon and Haak 2002).

The canines were not rewarded for positive indications after the first and second line-up identifications (see the diagram in Fig. 2) to not encourage the canine to indicate the same sample again.

All of the canines used in the experiments were specially trained for human scent identification. Their work experience ranged from 3 to 7 years.

\section{Statistical evaluations}

The results from the scent identification line-ups listed in Tables 3 and 4 were evaluated using Fisher's exact test and Pearson's chi-squared test by means of SAS 9.4. software (SAS Institute Inc. 2013. Base SAS ${ }^{\bullet} 9.4$ [computer program]. Guide: Statistical Procedures). Pearson's chi-squared test with 2 degrees of freedom was used for an association assessment between the different scent fractions and the correct canine identifications. 


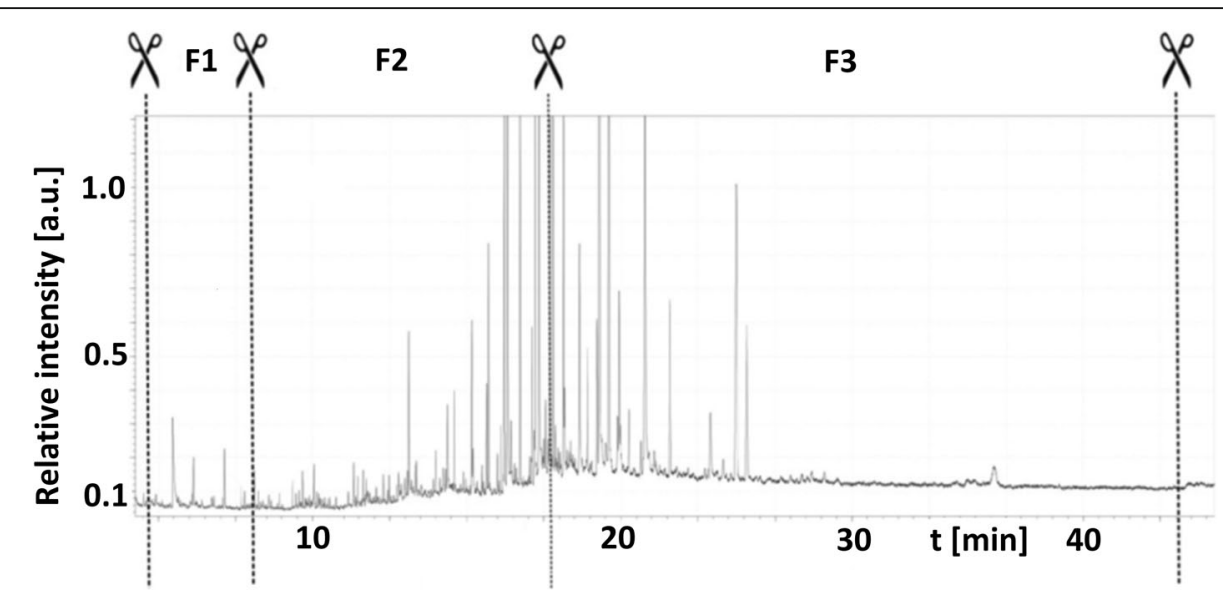

Fig. 1 Chromatogram of a human scent sample with the indicated intervals of the fractions

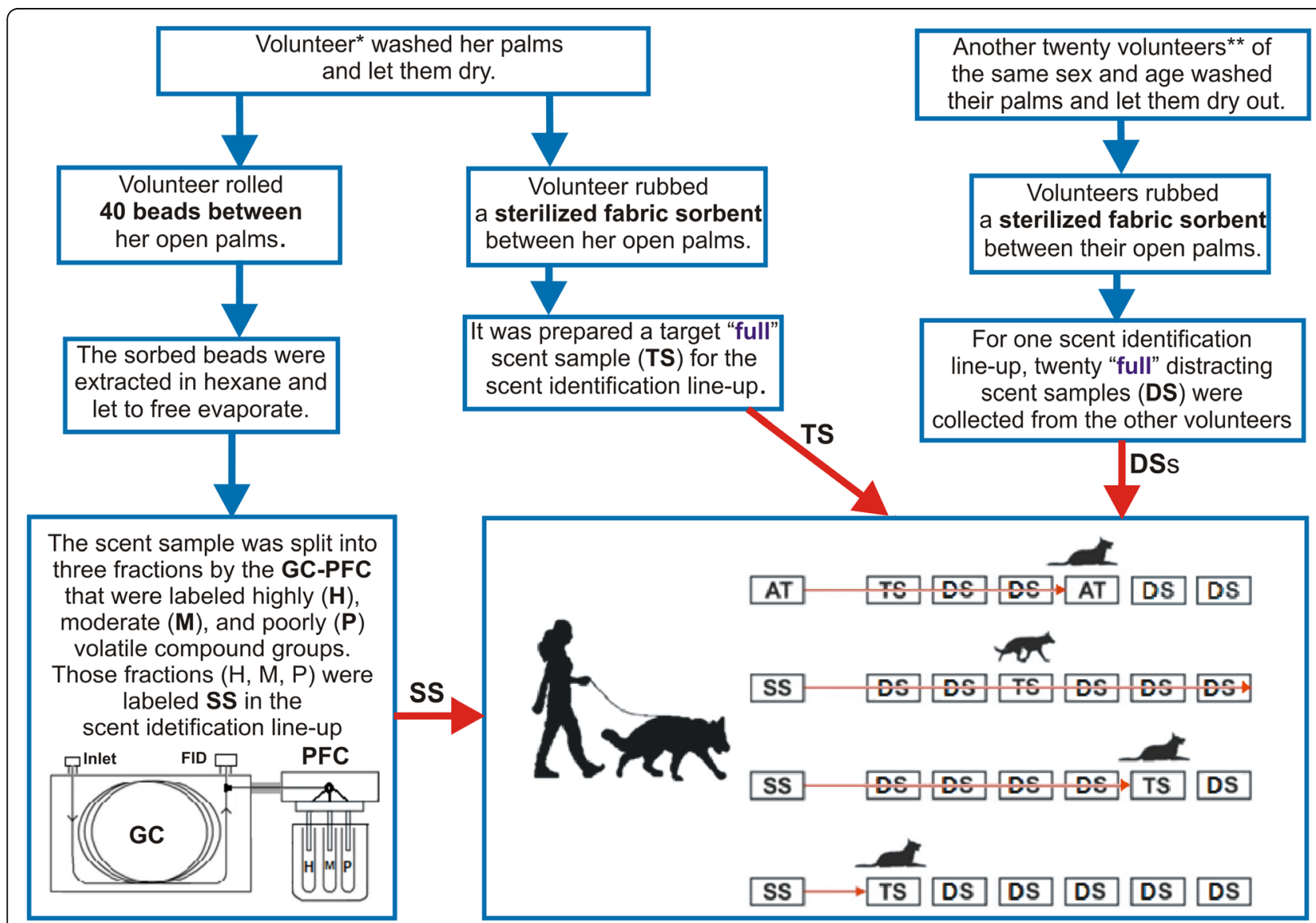

Fig. 2 Diagram of the collection, preparation, and testing of the human scent samples. The symbol SS denotes a starting scent represented by one fraction, TS denotes a "full" target scent sample (see text above), and AT denotes a scent sample used for an "attractivity test" (see text). In the diagram, volunteer* denotes one of the 11 volunteers whose scent samples were fractioned. The designation volunteers ${ }^{* *}$ refers to the group of 20 other volunteers who provided their scent samples. These samples were used as the distracting scents (DSs) 
Table 3 Results of the human scent identification line-ups performed using six canines

\begin{tabular}{llll}
\hline Volunteer & F1 & F2 & F3 \\
\hline 1 & $-++(\mathrm{A} 4)$ & $-++(\mathrm{C} 3)$ & $+++(\mathrm{D} 6)$ \\
2 & $+++(\mathrm{D} 6)$ & $+++(\mathrm{A} 4)$ & $+++(\mathrm{C} 3)$ \\
3 & $++-(\mathrm{A} 4)$ & $---(\mathrm{C} 3)$ & $+-+(\mathrm{E} 6)$ \\
4 & $---(\mathrm{B} 5)$ & $---(\mathrm{B} 5)$ & $+++(\mathrm{B} 5)$ \\
5 & $---(\mathrm{B} 5)$ & $---(\mathrm{B} 5)$ & $+++(\mathrm{B} 5)$ \\
6 & $---(\mathrm{B} 5)$ & $+++(\mathrm{D} 6)$ & $-++(\mathrm{B} 5)$ \\
7 & $---(\mathrm{D} 6)$ & $-++(\mathrm{B} 5)$ & $+++(\mathrm{D} 6)$ \\
8 & $--+(\mathrm{B} 5)$ & $+++(\mathrm{D} 6)$ & $+++(\mathrm{C} 3)$ \\
9 & $+++(\mathrm{C} 3)$ & $+++(\mathrm{E} 6)$ & $+++(\mathrm{F} 7)$ \\
10 & $---(\mathrm{C} 3)$ & $---(\mathrm{E} 6)$ & $+++(\mathrm{C} 3)$ \\
11 & $---(\mathrm{F} 7)$ & $+++(\mathrm{F} 7)$ & $---(\mathrm{E} 6)$
\end{tabular}

An individual volunteer (\#) in the table represents one scent sample that was split into three fractions (F1-F3) by the GC-PFC. A plus (+) means that the fraction was identified by the trained canine, a minus $(-)$ means the fraction was unidentified, and the results of the three trials are shown for each line-up. The letters $(A, B, C, D, E, F)$ with the numbers (3-7) in parentheses denote the individual canines with their corresponding line-up experience (from 3 to 7 years), respectively

\section{Results and discussion}

All of the results obtained from the human scent identifications using specially trained canines are summarized in Table 3. In these line-up identifications, the trained canines compared the fractioned starting scent (SS) samples with the "full" target samples (TS) and other distracting scents (DS) in the line-up.

Before scent identification, the same scent samples were analyzed by comprehensive two-dimensional gaschromatography-time of flight mass spectrometry (GCxGC-TOFMS) and the results were discussed in our study (Doležal et al. 2017). The partition of the individual fractions was based on this previous research. In this manner, the following groups of compounds were obtained:

The first scent fraction (see Fig. 1) contains highly volatile molecules (Dormont et al. 2013; Gallagher et al. 2008; Prada et al. 2015), e.g., volatile hydrocarbons, alcohols, aldehydes, aromatic compounds, and organic acid esters (up to approximately 7 carbons). The second fraction contains moderately volatile compounds such as hydrocarbons, alcohols, acids with longer chains, and fatty acid esters with shorter carbon chains. The third fraction contains poorly volatile compounds such as

Table 4 Success rate of the particular scent fractions in the scent identification line-up

\begin{tabular}{llll}
\hline Fraction & Identified & Unidentified & Number of trials \\
\hline 1st & 11 & 22 & 33 \\
2nd & 19 & 14 & 33 \\
3rd & 28 & 5 & 33 \\
\hline
\end{tabular}

fatty acid esters, mostly with $\mathrm{C}_{16}-\mathrm{C}_{18}$, different types of sterols, and certain vitamins.

The results obtained using the trained canines provided several remarkable observations. The results in Table 3 prove that a single human scent trace may contain several groups of molecules that make individual scent identifications possible. There are several scent signatures in one scent trace. In this paper, this phenomenon has been named the multiplicity of the human scent signature.

In addition to this finding, the results also show that the poorly volatile organic compounds (the 3rd fraction) are analogously as important for scent identification as the highly and moderately volatile compounds (the 1st and 2nd fractions). In our experiments, our specially trained canines most often preferred the poorly volatile molecules in the scent traces for the identification of people. Tables 3 and 4, which summarize the success rates for the scent identification line-ups for all the scent fractions, support this observation.

The poorly volatile fraction (the 3rd fraction) resulted in successful individual identification 28 times out of 33 attempts, thus an $85 \%$ success rate. The middle fraction (the 2nd fraction) resulted in successful identification 19 times out of $33(58 \%)$ and the highly volatile fraction (the 1st fraction) resulted in successful identification in 11 attempts out of 33 (30\%).

The statistical evaluations showed a significant association between the correct identification line-ups by canines and the particular scent fractions. There are highly significant differences in scent identification success according to the different scent fractions $(P<0.01)$. This observation is also supported by Pearson's chi-squared tests that unambiguously show that the correct identification line-ups depend with $99 \%$ certainty on the scent fraction used $(P<0.01)$. If all the scent identification results were taken together, the 3rd fraction was most often matched correctly (chi-square $=18.07,2$ degrees of freedom, $P<0.001)$. Considering the fraction couples separately, the 2nd and 3rd fractions were significantly more frequently correctly identified than the 1 st fraction. A highly significant difference was found between the scent identification successes of the 1 st and the 3rd fractions (chi-square $=11.20, P<0.01$ ). On the other hand, a less significant difference was found between the success rates of the 2 nd and the 3rd fractions (chi-square $=4.13$, $P<0.05)$, followed by the 1 st and the 2 nd fractions (chi-square $=2.71, P<0.05)$.

\section{Conclusions}

During this study, 198 "full" scent samples were collected from volunteers and used in line-up identifications as target (TS) and distracting scent (DS) samples (see diagram, Fig. 2). In addition, another 11 samples were collected on glass beads (see the "Methods" section). These scent 
samples were split into three disjointed fractions; in sum, 33 fractioned scent samples were prepared (3 for each volunteer), which were used as the starting samples (SS) in the line-up procedures. In total, 99 scent identifications were performed with 58 positive identifications $(+$, see Tables 3 and 4), and the remaining scent tests were performed without any identification (-). These results mean that the canines did not carry out any false identification!

The comparisons of the particular disjointed fractions with the "full" target scents (TS) and distracting scents (DS) in the line-ups using the trained canines provided two important findings.

The first finding is the observation that the human scent trace contains more than one group of compounds that allow for the individual scent identification of persons. This means that several human scent signatures co-exist in one scent trace. The canines may be performing these individual scent identifications in an analogous way to people identifying each other by sight. A human is able to identify a person based on his or her face, body posture, style of movement, hairstyle, etc.

Analogously, a canine can use different groups of molecules for the identification of individual people depending on various conditions. In both these cases, the different recognition methods (the different scent signatures) are variable in its reliability and durability.

The second principal observation is that the poorly volatile compounds in the human scent trace play a very important role in the scent identification procedure. Our experiments imply that their role is even more important than that of the highly and moderately volatile organic compounds. These volatile organic compounds are assumed to be the essential constituents of the human scent trace (Curran et al. 2010; Prada et al. 2015), and they are assumed to form the human scent signature. However, the obtained results could be influenced by the temperature program $\left(110-320^{\circ} \mathrm{C}\right.$ for $\left.46 \mathrm{~min}\right)$ during the fraction preparations by GC-PFC. Such conditions could cause the degradation of some thermolabile compounds and thus influence the molecular compositions of the individual fractions. This degradation may be the main factor reducing the success rates of the first and second fractions. Nevertheless, our experiments unambiguously confirm the multiplicity of the human scent signature and the significance of the poorly volatile compounds (as fatty acid esters) in the scent identification method.

We hope that the results of this study will open a discussion about the multiplicity of the human scent signature, primary scent molecules, optimal canine training, and in general, the scent identification method.

\section{Abbreviations}

A, B, C, D, E, F: Dogs; AT: Attractivity test; C16-C18: Hydrocarbons chain

DS: Distracting scent samples; F1-F3: Fractions 1-3; GC: Gas chromatography;
GC-PFC: Gas chromatograph connected with a preparative fraction collector; HS-SPME-GC/MS: Headspace solid-phase microextraction gas chromatography/ mass spectrometry; N2: Liquid nitrogen; No.: Number; PTV: Programmable temperature vaporization; RI: Retention index; SS: Starting scents sample; SSL: Splitless mode; SSL-PTV: Splitless mode-Programmable temperature vaporization; TS: Target scent sample; VOC: Volatile organic compounds

\section{Acknowledgements}

Not applicable.

\section{Funding}

This work was supported by the Ministry of the Interior of the Czech Republic [projects No. Vf20142016036, No. VI20172020075] and by the Institute of Organic Chemistry and Biochemistry of the CAS [research program RVO: 61388963]. This work was also supported by the project OPPC CZ.2.16/3.1.00/21537 and the project NPU I LO1601. The funders had no role in study design, data collection and analysis, decision to publish, or preparation of the manuscript

\section{Availability of data and materials}

The dataset(s) supporting the conclusions of this article is (are) included within the article (and its additional file(s)).

\section{Authors' contributions}

PD, KGF, and ŠU suggested project conceptions, study ideas, and experimental designs. PD, IV, and PK performed the GC-PFC analyses. JL and LP performed all the scent identification line-up experiments, and they were responsible for the canine trainings. VŠ and PD collected the human scent sample from the volunteers, and they organized their recruitment. All the co-authors discussed the results together. $\breve{U}$ and PD prepared the article text. All authors read and approved the final manuscript.

\section{Ethics approval and consent to participate}

This study was approved by the Institutional Review Board of Charles University, Praque, Faculty of Science (approval number 2016/28) and complies with the Declaration of Helsinki for Medical Research involving Human Subjects. The recruited volunteers were verbally instructed regarding the sampling method and analysis, and they each signed an agreement (written consent) regarding the use of their scent samples in this experiment. The samples from volunteers were used for this experiment only, and then these samples were destroyed.

This study also strictly observed Act No. 246/1992 Coll. as subsequently amended "On the protection of animals against cruelty", and the protocol was approved by the Expert Commission for the Welfare of Experimental Animals of the Czech University of Life Sciences Prague under Resolution No. 01/14 on 3rd February 2014. The Canine Behavior Research Center was approved by the Ministry of Agriculture of the Czech Republic to perform experiments on canines under Resolution No. 16OZ13147/2013-171,241. The handling of the canines did not involve the routine training of the canines for the scent identification procedure.

The Ethics approval was attached in this manuscript submission.

\section{Consent for publication}

Not applicable.

\section{Competing interests}

The authors declare that they have no competing interests.

\section{Publisher's Note}

Springer Nature remains neutral with regard to jurisdictional claims in published maps and institutional affiliations.

\section{Author details}

${ }^{1}$ Department of Analytical Chemistry, University of Chemistry and Technology Prague, Prague 6, Czech Republic. ${ }^{2}$ International Forensic Research Institute, Department of Chemistry and Biochemistry, Florida International University, Miami, USA. ${ }^{3}$ Canine Behavior Research Center, Department of Animal Science and Ethology, Czech University of Life Sciences Prague, Prague 6, Czech Republic. ${ }^{4}$ Institute of Organic Chemistry and Biochemistry, Czech Academy of Sciences, Prague 6, Czech Republic. 
Received: 8 October 2018 Accepted: 29 January 2019

Published online: 12 February 2019

\section{References}

Bernier UR, Booth MM, Yost RA (1999) Analysis of human skin emanations by gas chromatography mass spectrometry. 1. Thermal desorption of attractants for the yellow fever mosquito (Aedes aegypti) from handled glass beads. Anal Chem 71:1-7

Cui SF, Tan S, Ouyang GF, Jiang SH, Pawliszyn J (2009) Headspace solid-phase microextraction gas chromatography-mass spectrometry analysis of Eupatorium odoratum extract as an oviposition repellent. J Chromatogr B 877:1901-1906

Curran AM, Prada PA, Furton KG (2010) The differentiation of the volatile organic signatures of individuals through SPME-GC/MS of characteristic human scent compounds. J Forensic Sci 55:50-57

Curran AM, Rabin SI, Furton KG (2005) Analysis of the uniqueness and persistence of human scent. Forensic Sci Commun 7:1-20

Doležal P, Kyjaková P, Valterová I, Urban Š (2017) Qualitative analyses of less-volatile organic molecules from female skin scents by comprehensive two dimensional gas chromatography-time of flight mass spectrometry. J Chromatogr A 1505:77-86

Dormont L, Bessière J-M, McKey D, Cohuet A (2013) New methods for field collection of human skin volatiles and perspectives for their application in the chemical ecology of human-pathogen-vector interactions. J Exp Biol 216:2783-2788

Gallagher M, Wysocki CJ, Leyden JJ, Spielman Al, Sun X, Preti G (2008) Analyses of volatile organic compounds from human skin. Br J Dermatol 159:780-791

Jezierski T, Sobczynska M, Walczak M, Gorecka-Bruzda A, Ensminger J (2012) Do trained dogs discriminate individual body odors of women better than those of men? J Forensic Sci 57:647-653

Kusano M, Mendez E, Furton KG (2011) Development of headspace SPME method for analysis of volatile organic compounds present in human biological specimens. Anal Bioanal Chem 400:1817-1826

Marchal S, Bregeras O, Puaux D, Gervais R, Ferry B (2016) Rigorous training of dogs leads to high accuracy in human scent matching-to-sample performance. PLoS One 11:e0146963

Pandey SK, Kim KH (2011) Human body-odor components and their determination. Trac-Trend Anal Chem 30:784-796

Pinc L, Bartoš L, Reslová A, Kotrba R (2011) Dogs discriminate identical twins. PLoS One 6:e20704

Prada PA, Curran AM, Furton KG (2010) Comparison of extraction methods for the removal of volatile organic compounds (VOCs) present in sorbents used for human scent evidence collection. Anal Methods 2:470-478

Prada PA, Curran AM, Furton KG (2015) Human Scent Evidence. CRC Press, Taylor \& Francis Group, Boca Raton

Santariová M, Pinc L, Bartoš L, Vyplelová P, Gerneš J, Sekyrová V (2016) Resistance of human odours to extremely high temperature as revealed by trained dogs. Czech J Anim Sci 61:172-176

SAS Institute Inc. 2013. Base SAS ${ }^{\oplus}$ 9.4. Guide: Statistical Procedures nE, Cary, NC, USA

Schoon A, Haak R (2002) K9 suspect discrimination: training and practicing scent identification line-ups. Detselig Enterprises, Calgary

Schoon GAA (1996) Scent identification lineups by dogs (Canis familiaris): experimental design and forensic application. Appl Anim Behav Sci 49:257-267

Schoon GAA (2005) The effect of the ageing of crime scene objects on the results of scent identification line-ups using trained dogs. Forensic Sci Int 147:43-47

Settle RH, Sommerville BA, McCormick J, Broom DM (1994) Human scent matching using specially trained dogs. Anim Behav 48:1443-1448

Tomaszewski T, Girdwoyn P (2006) Scent identification evidence in jurisdiction (drawing on the example of judicial practice in Poland). Forensic Sci Int 162:191-195

Vyplelová P, Vokálek V, Pinc L, Pacáková Z, Bartoš L, Santariová M, Čapková Z (2014) Individual human odor fallout as detected by trained canines. Forensic Sci Int 234:13-15

\section{Submit your manuscript to a SpringerOpen ${ }^{\circ}$ journal and benefit from:}

- Convenient online submission

- Rigorous peer review

- Open access: articles freely available online

- High visibility within the field

- Retaining the copyright to your article

Submit your next manuscript at $\boldsymbol{\nabla}$ springeropen.com 